Pacific Journal of Mathematics

ON EXTREMAL PROPERTIES FOR ANNULAR RADIAL AND
CIRCULAR SLIT MAPPINGS OF BORDERED RIEMANN

U UL ADrian NICKEL 


\section{ON EXTREMAL PROPERTIES FOR ANNULAR RADIAL AND CIRCULAR SLIT MAPPINGS OF BORDERED RIEMANN SURFACES}

\section{Paul A. Nickel}

Introduction. There exist functions which map a planar Riemann surface $W$ of arbitrary conectivity conformally onto plane slit regions. Functionals $I$, extremized in the class of all conformal mappings of $W$ by only one slit mapping, are known. Such functionals can be represented as limits of functionals $I_{n}$, where each $I_{n}$ is itself extremized by a horizontal or vertical-slit mapping with domain of finite connectivity.

A planar bordered Riemann surface of finite connectivity can be mapped conformally onto a radial or circular-slit annulus with inner and outer boundaries corresponding to any two contours of the surface. In this investigation, extremal properties of such mappings are obtained and extended to surfaces of infinite connectivity. The geometric nature of the extended mappings, called principal analytic functions, is then deduced from the extended extremal properties. In addition, certain combinations of principal analytic functions are investigated from both extremal and geometric points of view.

First, we consider a planar bordered oriented Riemann surface $\bar{W}$, of infinite connectivity. It is assumed that $\bar{W}$ has two compact border components, $\delta$ and $\gamma$, such that no point of $\delta \cup \gamma$ is a limit point of points of any other boundary components. Such contours are called isolated. $\bar{W}$ is "approximated" by a sequence of compact bordered Riemann surfaces $\left\{W_{n}\right\}$, where each $W_{n}$ is of finite connectivity. On $W_{n}$, annular radial and circular-slit mappings $F_{0 n}$ and $F_{1 n}$ are constructed. Among all normalized conformal annular mappings $F$ of $W_{n}, F_{0 n}$ maximizes

$$
2 \pi \log (r(F))+\mu_{n}(F)
$$

and $F_{1 n}$ minimizes

$$
2 \pi \log (r(F))-\mu_{n}(F) .
$$

Here, $r(F)$ is the quotient $r_{\gamma} / r_{\delta}$, where $r_{\gamma}$ and $r_{\delta}$ represent the radii of the positively oriented $F(\gamma)$ and the negatively oriented $F(\delta)$ respectively, and $\mu_{n}(F)$ is the complementary area of $\log \left(F\left(W_{n}\right)\right)$.

It is then shown by the reduction theorem (Sario[4]) that these extremal properties hold in the limit for the limit functions $F_{0}$ and $F_{1}$.

Received August 22, 1960. The results of this paper are part of the contents of the author's Ph. D. thesis, done under the direction of Professor Leo Sario, to whom I wish to express my most sincere gratitude. 
Furthermore, the extremal properties of $F_{0}$ and $F_{1}$ imply that the former is a radial slit mapping of $\bar{W}$ and that the latter is a circular slit mapping. By establishing a deviation formula, it is seen that the functions $F_{0}$ and $F_{1}$ are, up to a rotation, the only normalized conformal annular maps of $\bar{W}$ extremizing the limit functionals. As another application of the reduction theorem, we find that the univalent function $\sqrt{F_{0} \cdot F_{1}}$ maximizes $\mu(F)$, the complementary logarithmic area, among all conformal annular mappings of $\bar{W}$.

Next we pose the question: When does $\bar{W}$ have distinct radial and circular-slit mappings. The answer is given in terms of $A D$-removability, at least when $\bar{W}$ is a plane region bounded by an outer contour $\gamma$ and an inner contour $\delta$. A point set $E$ of the extended plane is called $A D$ removable when the only analytic functions with finite Dirichlet integral, defined on the complement of $E$, are the constant functions. In particular, we find that the principal analytic functions are, up to a rotation, identical, if and only if the plane region bounded by $\gamma$ and $\delta$ minus $\bar{W}$ is $A D$-removable.

1. We consider $\bar{W}$ an open planar bordered Riemann surface with two compact non-point border components, $\delta$ and $\gamma$. In order to describe the remaining part of the boundary of $\bar{W}$, we recall that such a surface can be embedded in a Riemann sphere $S^{2}$. With respect to this embedding, we assume that $\bar{W}$ and its boundary components satisfy the following conditions:

(1) no point of $\delta \cup \gamma$ is a limit point of points of any other boundary components, and (2) $\bar{W}-(\delta \cup \gamma)$ is open in $S^{2}$. Operations in $\bar{W}$ such as interior, boundary, etc., are referred to $S^{2}$.

It is possible to exhaust an open Riemann surface by a countable collection of compact approximating regions $\left\{W_{n}\right\}$. In fact, $\bar{W}$ can be countably exhausted in the following modified sense:

1. $\delta \cup \gamma \subset W_{n}$.

2. $W_{n} \subset \operatorname{Int} W_{n+1}$.

3. The boundary of $W_{n}$ consists of a finite number of disjoint analytic Jordan curves.

4. Each component of $\bar{W}-W_{n}$ is relatively non-compact.

5. $\bar{W}=\cup W_{n}$.

There is no loss in generality in assuming that each $W_{n}$ contains a $\zeta \in \bar{W}$, where $\zeta$ is arbitrary but fixed in advance.

Evidently $\delta$ and $\gamma$ are two border components of $W_{n}$. The remaining border components will be denoted $\beta_{1}\left(W_{n}\right), \beta_{2}\left(W_{n}\right), \cdots, \beta_{k(n)}\left(W_{n}\right)$. When only one approximating subregion is under consideration, the notation for these remaining border components will be shortened to $\beta_{1}, \beta_{2}, \cdots$, $\beta_{k(n)}$. For convenience we define $\bar{\beta}_{n}$ as 


$$
\bar{\beta}_{n}=\cup \beta_{i}\left(W_{n}\right) . \quad i=1,2, \cdots k(n)
$$

\section{Extremal Properties of Harmonic Functions Defined on Approxi- mating Regions.}

2. We consider, in this and the following section, certain classes of harmonic and analytic functions defined on an approximating region $W_{n}$.

Definition. $H_{n}(h+k)$ is the set of functions $p$, harmonic on Int $W_{n} \cup \delta \cup \gamma$ and satisfying

(1) $p(z)=c_{2}(p)=$ const. for $z \in \gamma$ with $\int_{\gamma} d p^{*}=2 \pi(h+k)$,

(2) $p(\zeta)=0$,

(3) $p(z)=c_{1}(p)$ for $z \in \delta$ with $\int_{\delta} d p^{*}=-2 \pi(h+k)$, and

(4) $\int_{\beta_{i}} d p^{*}=0$ for $i=1,2, \cdots, k(n)$.

$h$ and $k$ are real numbers. When the function $p$ is defined only on Int $W_{n} \cup \delta \cup \gamma$, then the integrals $\int_{\beta_{i}\left(W_{n}\right)} d p^{*}$ and $\int_{\beta_{i}\left(W_{n}\right)} p d p^{*}$ are understood as $\lim _{k \rightarrow \infty} \int_{\beta_{i}\left(W^{\prime} k^{\prime}\right)} d p^{*}$ and $\lim _{k \rightarrow \infty} \int_{\beta_{i}\left(W^{\prime} k^{k}\right)} p d p^{*}$. Here $\left\{W_{k}^{\prime}\right\}$ is an exhaustion of the surface Int $W_{n}$ and each $\beta_{i}\left(W_{k}^{\prime}\right)$ is homologous (in $\left.W_{n}\right)$ to $\beta_{i}\left(W_{n}\right)$. An application of Green's formula shows that these limits are independent of the exhaustion $\left\{W_{k}^{\prime}\right\}$. The class $H_{n}(1)$ will be denoted $H_{n}$.

Principal harmonic functions $p_{0 n}$ and $p_{1 n}$, belonging to $H_{n}$ are obtained as harmonic extensions of functions constructed by use of linear operators on Riemann surfaces (Sario [2]). In fact on each $\beta_{i}, i=1,2, \cdots, k(n)$, $\partial p_{0 n} / \partial n=0$ and $p_{1 n}=$ const. Hence for arbitrary $h$ and $k$, the function $p_{h k n}=h p_{0 n}+k p_{1 n}$ belongs to the class $H_{n}(h+k)$, which is then not empty.

3. THeorem 1. $P_{h k n}$ minimizes the functional $\int_{\bar{\beta}_{n}} p d p^{*}-$ $2 \pi(h-k) c(p)$ among all $p \in H_{n}(h+k)$, where $c(p)=c_{2}(p)-c_{1}(p)$.

The value of the minimum is $-2 \pi\left[h^{2} c\left(p_{0 n}\right)-k^{2} c\left(p_{1 n}\right)\right]$.

The deviation of this functional from its minimum is $D_{W_{n}}\left(p-p_{k k n}\right)$, and the minimizing function is unique.

Proof. Let $B$ be the entire border of $W_{n}$. Then by Green's formula, we have

$$
D_{W_{n}}\left(p-p_{h k n}\right)=\int_{B}\left(p-p_{h k n}\right) d\left(p-p_{h k n}\right)^{*} .
$$

Since $p$ and $p_{h k n} \in H_{n}(h+k)$, we conclude at once that $\int_{\delta+\gamma}\left(p-p_{h k n}\right)$ $d\left(p-p_{h k n}\right)^{*}=0$. Green's formula becomes 


$$
D_{W_{n}}\left(p-p_{h k n}\right)=\int_{\bar{\beta}_{n}} p d p^{*}+\int_{\bar{\beta}_{n}} p_{h k n} d p_{h k n}^{*}-\int_{\bar{\beta}_{n}} p_{h k n} d p^{*}+p d p_{h k n}^{*} .
$$

We now expand the last term and find that

$$
\int_{\bar{\beta}_{n}} p_{h k n} d p^{*}+p d p_{h k n}^{*}=h \int_{\bar{\beta}_{n}} p_{o n} d p^{*}+p d p_{o n}^{*}+k \int_{\bar{\beta}_{n}} p_{1 n} d p^{*}+p d p_{1 n}^{*} .
$$

But on $\bar{\beta}_{n}, p_{0 n}$ has vanishing normal derivative, and $p_{1 n}$ is constant. This means that $\int_{\bar{\beta}_{n}} p d p_{0 n}^{*}=\int_{\bar{\beta}_{n}} p_{1 n} d p^{*}=0$ when $p \in H_{n}(h+k)$. Thus we can infer from Green's formula that

$$
\int_{\bar{\beta}_{n}} p_{h k n} d p^{*}+p d p_{h k n}^{*}=h \int_{\delta+\gamma} p_{0 n} d p^{*}-p d p_{0 n}^{*}+k \int_{\delta+\gamma} p_{1 n} d p^{*}-p d p_{1 n}^{*} .
$$

A direct application of the conditions (1), (3), (4) of $H_{n}(h+k)$ now yields the formula

$$
\begin{gathered}
\int_{\bar{\beta}_{n}} p_{h k n} d p^{*}+p d p_{h k n}^{*}=2 \pi(h-k)\left(c_{2}(p)-c_{1}(p)\right) \\
-2 \pi h(h+k)\left(c_{2}\left(p_{0 n}\right)-c_{1}\left(p_{0 n}\right)\right) \\
+2 \pi k(h+k)\left(c_{2}\left(p_{1 n}\right)-c_{1}\left(p_{1 n}\right)\right) .
\end{gathered}
$$

We obtain in a similar fashion

$$
\begin{aligned}
\int_{\bar{\beta}_{n}} p_{h k n} d p_{h k n}^{*} & =h k \int_{\bar{\beta}_{n}} p_{0 n} d p_{1 n}^{*}-p_{1 n} d p_{0 n}^{*} \\
& =-h k \int_{\delta+\gamma} p_{0 n} d p_{1 n}^{*}-p_{1 n} d p_{0 n}^{*} \\
= & -2 \pi h k\left[c_{2}\left(p_{0 n}\right)-c_{1}\left(p_{0 n}\right)-\left(c_{2}\left(p_{1 n}\right)-c_{1}\left(p_{1 n}\right)\right)\right] .
\end{aligned}
$$

Collecting contributions, we find

$$
\begin{gathered}
D_{W_{n}}\left(p-p_{h k n}\right)-2 \pi\left[h^{2}\left(c_{2}\left(p_{0 n}\right)-c_{1}\left(p_{0 n}\right)\right)-k^{2}\left(c_{2}\left(p_{1 n}\right)-c_{1}\left(p_{1 n}\right)\right)\right] \\
=\int_{\bar{\beta}_{n}} p d p^{*}-2 \pi(h-k)\left(c_{2}(p)-c_{1}(p)\right) .
\end{gathered}
$$

Since the Dirichlet integral is nonnegative, we have that $p_{h k n}$ minimizes the given functional. Clearly, for any $p \in H_{n}(h+k)$ the deviation of the functional from its minimum is $D_{W_{n}}\left(p-p_{h k n}\right)$.

We consider now the uniqueness of the minimizing function. For another minimizing function $p^{\prime}$, we would have a deviation of the functional from the minimum equal to $D_{\mathrm{w}_{n}}\left(p^{\prime}-p_{h k n}\right)$. But $p^{\prime}$ also minimizes, so $D_{W_{n}}\left(p^{\prime}-p_{h k n}\right)=0$. Since $p_{h k n}(\zeta)=p^{\prime}(\zeta)=0$, we see that $p_{h k n}=p^{\prime}$. This completes the proof of Theorem 1.

4. Our interest in Theorem 1 will be with the following special cases which we state as corollaries. 
COROLLARY 1. $p_{0 n}$ maximizes the functional $2 \pi c(p)-\int_{\bar{\beta}_{n}} p d p^{*}$ among all $p \in H_{n}$.

COROLlaRY 2. $p_{1 n}$ minimizes the functional $2 \pi c(p)+\int_{\bar{\beta}_{n}} p d p^{*}$ among all $p \in H_{n}$.

COROLlaRY 3. $\frac{1}{2}\left(p_{0 n}+p_{1 n}\right)$ minimizes the functional $\int_{\bar{\beta}_{n}} p d p^{*}$ among all $p \in H_{n}$.

Corollary 4. $p_{0 n}-p_{1 n}$ maximizes the functional $4 \pi c(p)-D_{W_{n}}(p)$ among all $p \in H_{n}(0)$.

Each extremizing function is unique.

Corollaries 1, 2, and 3 follow immediately from Theorem 1 for $h+k=1$. As for Corollary 4, clearly $p_{0 n}-p_{1 n} \in H_{n}(0)$. Now for any $p \in H_{n}(0)$, Green's formula reads $D_{W_{n}}(p)=\int_{\gamma+\delta+\bar{\beta}_{n}} p d p^{*}=\int_{\bar{\beta}_{n}} p d p^{*}$, and Corollary 4 follows.

II. Geometric Properties of Analytic Functions Defined on Approximating Regions.

5. Definition. $A_{n}$ is the class of functions $F$ analytic on Int $W_{n} \cup \delta \cup \gamma$ such that

(1) $F(\gamma)$ is a circle traced once in the positive direction,

(2) $|F(\zeta)|=1$,

(3) $F(\delta)$ is a circle traced once in the negative direction,

(4) $F$ is univalent on Int $W_{n} \cup \delta \cup \gamma$.

In this definition, $F(\gamma)$ and $F(\delta)$ are understood as oriented images of oriented border cycles and the radii of these images are denoted $r_{\gamma}\left(F^{\prime}\right)$ and $r_{\delta}(F)$.

Some useful relations between the classes $A_{n}$ and $H_{n}$ are expressed in the following theorem.

6. Theorem 2. (a) For any $F \in A_{n}, \log |F|$ is of class $H_{n}$.

(b) The following analytic functions are of class $A_{n}$ :

$$
F_{0 n}=\exp \left(p_{0 n}+i p_{0 n}{ }^{*}\right),
$$$$
\text { (2) } F_{1 n}=\exp \left(p_{1 n}+i p_{1 n}{ }^{*}\right) \text {. }
$$

The functions $F_{i n}$ are referred to as principal analytic functions.

Proof of $(a)$. Evidently $2 \pi=\int_{\gamma} d(\arg F(z))=\int_{\gamma} d(\log |F(z)|)^{*} \quad$ and 
Condition 1 of $H_{n}$ is verified. Condition 3 is checked just as easily and (2) is apparent. As for (4), let $\beta_{i}$ be any component of the border of $W_{n}$ other than $\delta$ or $\gamma$. Suppose that $\beta_{i}^{\prime} \sim \beta_{i}$ and that $\int_{\beta_{i}} d(\log |F|)^{*}=$ $2 \pi k$, where $k$ is an integer. There exists a path from $\delta$ to $\gamma$ which does not meet $\beta_{i}^{\prime}$. But if $k \neq 0$, then every path from $F(\delta)$ to $F(\gamma)$ meets $F\left(\beta_{i}^{\prime}\right)$. But $F$ is univalent, so $k=0$.

Proof of $(b)$. We consider first the function $F_{1 n}$ and omit the analogous proof for $F_{0 n}$. First, it is evident that $2 \pi=\int_{\gamma} d p_{1 n}^{*}=\int_{\gamma} d\left(\arg F_{1 n}\right)$ and $r_{\gamma}(F)=\exp c_{2}\left(p_{1 n}\right)=$ const. Certainly $F_{1 n}(\gamma)$ is a circle traced once in the positive direction, and (1) of No. 5 is satisfied. Condition 3 is verified in a similar manner and (2) is trivial.

To verify the Condition 4, we consider the extended version of the argument principle, and reason in a manner analogous to Ahlfors [1], p. 203.

7. Definition. The multiple-valued functions $P_{i n}$ are defined as $P_{i n}=p_{i n}+i p_{i n}^{*}$. However $P_{0 n}-P_{1 n}$ is single-valued, and the principal analytic functions are expressible as $F_{i n}=\exp P_{i n}, i=0,1$. We also fix the following terminology: $r(F)$ denotes the ratio $r_{\gamma}(F) / r_{\delta}(F)$ and $\mu_{n}(F)$ denotes the complementary logarithmic area $-\int_{\bar{\beta}_{n}} \log |F(z)| d(\arg F(z))$, a nonnegative quantity when $F \in A_{n}$.

THEOREM 3. $F_{0 n}$ maximizes $2 \pi \log r(F)+\mu_{n}(F)$ among all $F \in A_{n}$. $F_{1 n}$ minimizes $2 \pi \log r(F)-\mu_{n}(F)$ among all $F \in A_{n}$.

$P_{n}=\sqrt{F_{0 n} \cdot F_{1 n}}$ maximizes $\mu_{n}(F)$ among all $F \in A_{n}$.

$F_{0 n} / F_{1 n}$ maximizes $4 \pi \log r(F)-D_{W_{n}}(\log |F|)$ among all quotients of functions in $A_{n}$.

$P_{0 n}-P_{1 n}$ maximizes $4 \pi\left[\operatorname{Re}\left(F\left(z_{2}\right)-F\left(z_{1}\right)\right)\right]-D_{W_{n}}(F)$ among all analytic functions on $W_{n}$ the real part of which is constant on $\delta$,constant on $\gamma$, and 0 at $\zeta$. Here $z_{2}$ and $z_{1}$ are on $\gamma$ and $\delta$ respectively.

Proof. We have $\log \left|F_{0 n}(z)\right|=p_{0 n}(z)$, so it follows from Corollary 1 of Theorem 1 that $\log \left|F_{0 n}\right|$ maximizes the functional $2 \pi c(p)-\int_{\bar{\beta}_{n}} p d p^{*}$ among all $p \in H_{n}$. But according to Theorem 2, when $F \in A_{n}$, the $\log |F(z)| \in H_{n}$. Hence $F_{0 n}$ maximizes the functional $2 \pi \log r(F)+\mu_{n}(F)$ among all $F \in A_{n}$. The proof of the second part of this theorem is analogous, and so is the proof of the third part when it is shown that $P_{n}=\sqrt{F_{0 n} \cdot F_{1 n}}$ is of class $A_{n}$, a fact that is proved in the appendix.

It is easily seen that $\log \left|F_{0 n}\right| F_{1 n} \mid=p_{0 n}-p_{1 n}$, hence according to Corollary 4 of Theorem $1, \log \left|F_{0 n}\right| F_{1 n} \mid$ maximizes $4 \pi c(p)-D_{\mathbb{v}_{n}}(p)$ 
among all $p \in H_{n}(0)$. If $\mathrm{F}=G / H$, where $G$ and $H \in A_{n}$, then it follows from Theorem 2 that $\log |G|$ and $\log |H| \in H_{n}$, and we have $\int_{\gamma} d(\log |F|)^{*}=$ $\int_{\gamma} d(\arg F)=\int_{\gamma} d(\arg G / H)=0$. Other similar calculations show that $\log |F(z)| \in H_{n}(0)$. Thus $F_{0 n} / F_{1 n}$ maximizes $4 \pi \log r(F)-D_{W_{n}}(\log |F|)$ among all quotients of functions in $A_{n}$.

The extremal property of $P_{0 n}-P_{1 n}$ follows from Corollary 4 as well when it is observed that $\operatorname{Re}\left(P_{0 n}-P_{1 n}\right)=p_{0 n}-p_{1 n}$, and that $\operatorname{ReF} \in H_{n}(0)$ when $F$ is analytic on $W_{n}$.

The following corollary of Theorem 3 will be useful when we are considering geometric properties of conformal maps of $\bar{W}$.

Corollary. The functional $r(F)$ is maximized, uniquely up to a rotation, by $F_{0 n}$ and minimized, uniquely up to a rotation, by $F_{1 n}$ among all $F \in A_{n}$.

Proof. It follows from the definition of $F_{0 n}$ given in No. 6. that $d\left(\arg F_{0 n}\right)=d p_{0 n}^{*}$, which is 0 on $\bar{\beta}_{n}$. Since $\mu_{n}(F) \geqq 0$, we have $2 \pi \log r(F) \leqq$ $2 \pi \log r(F)+\mu_{n}(F) \leqq 2 \pi \log r\left(F_{0 n}\right)+\mu_{n}\left(F_{0 n}\right)=2 \pi \log r\left(F_{0 n}\right)$, that is, $r(F)$ is maximized by $F_{0 n}$.

Analogous reasoning shows that $F_{1 n}$ minimizes $r(F)$ among all $F \in A_{n}$.

In order to establish the uniqueness, we let $r(F)=r\left(F_{0 n}\right)$ for some $F \in A_{n}$. Then an application of Theorem 3 yields $0<\mu_{n}(F) \leqq \mu_{n}\left(F_{0 n}\right) \leqq 0$, which means that $F$ also maximizes the functional $2 \pi \log r(F)+\mu_{n}(F)$ among $F \in A_{n}$. But an application of the deviation formula of Theorem 1 shows that $D_{w_{n}}\left(\log |F| F_{0 n} \mid\right)=0$, from which it follows that $F=c F_{0 n}$ with $|c|=1$.

\section{Extremal Properties of Principal Harmonic Functions.}

8. We propose in the present section, to develop for domains of infinite connectivity, extremal theorems which will generalize the results of $\S 1$ for finite connectivity. An essential role is played by the

Reduction Theorem (Sario [4]).

Assume that $Z$ and $Z_{n}$ are classes of functions with domains $W$, an arbitrary open Riemann surface, and $W_{n}$, an exhausting subregion of $W$, respectively. In addition, suppose that real-valued functionals $m$ and $m_{n}$, defined on $Z$ and $Z_{n}$, satisfy the following conditions.

(R1) If $W_{m} \subset W_{n}$ and if $f \in Z_{n}$, then $\left.f\right|_{W_{m}} \in Z_{m}$.

Here $W_{n}$ may be replaced by $W$, and $Z_{n}$ by $Z$.

(R2) If $\left\{f_{k}\right\}$ is a sequence the elements of which belong to $Z_{n}$, and if $\left\{f_{k}\right\}$ converges uniformly to $f \in Z_{n}$, then $m_{n}\left(f_{k}\right)$ converges to $m_{n}(f)$.

(R3) $m(f)=\lim _{n \rightarrow \infty} m_{n}(f)$, for any $f \in Z$. 
(R4) There exists a function $f_{n} \in Z_{n}$ such that $f_{n}$ minimizes the functional $m_{n}$ among all $f \in Z_{n}$.

(R5) For $k<h$, and $f \in Z_{h}, m_{k}(f) \leqq m_{h}(f)$.

(R6) The family $\left\{f_{n} ; f_{n}\right.$ minimizes $m_{n}$ among $\left.f \in Z_{n}\right\}$ is a normal family, and the limit functions belong to $Z$.

Then any limit function $f=\lim _{n \rightarrow \infty} f_{n}$ minimizes $m$ among all $f \in Z$, and value of minimum is $m(f)=\lim _{n \rightarrow \infty} m_{n}\left(f_{n}\right)$.

The proof of the reduction theorem is established by selecting an exhaustion of $W$, and can be carried out for a bordered surface $\bar{W}$ as well, as soon as an exhaustion is known to exist.

9. Let $\bar{W}$ be an open planar bordered Riemann surface, $\left\{W_{n}\right\}$ an exhausting set, $\delta$ and $\gamma$ separated boundary components, all as described is no. 1 .

Lemma 1. The families $\left\{p_{0 n}\right\}$ and $\left\{p_{1 n}\right\}$ are normal.

Proof. If $\left\{F_{0 n}\right\} \quad\left(\left\{F_{1 n}\right\}\right)$ is a normal family, then so is $\left\{p_{0 n}\right\}\left(\left\{p_{1 x}\right\}\right)$. Hence it suffices to show that for every compact set $S$, there exist a constant $M$ and and integer $N$ such that $\left|F_{0 n}(z)\right|<M\left(\left|F_{1 n}(z)\right|<M\right)$ for all $n>N$ and all $z \in S$. Let $S$ be any compact subset of $\bar{W}$ and choose $n$ sufficiently large so that $S \subset W_{n}$. For any $z \in S$ and $W_{p} \subset W_{n}$, since $F_{0 p}(\gamma)$ is the outer contour of an image annulus we have $2 \pi \log \mid F_{0 p}(z) /$ $r_{\delta}\left(F_{0 p}\right) \mid \leqq 2 \pi \log \left(r\left(F_{0 p}\right)\right)+\mu_{n}\left(F_{0 p}\right)$. But according to Theorem 3 , the right hand side is bounded by $2 \pi \log \left(r\left(F_{0 n}\right)\right)$. We now recall that $\left|F_{0 p}(\zeta)\right|=1$, that is $r_{\delta}\left(F_{0 p}\right)<1$. Hence $\left|F_{0 p}(z)\right|$ is bounded for all $z \in S$ and for all $p \geqq n$, and the family $\left\{F_{0 n}\right\}$ is normal.

As for $\left\{F_{1 n}\right\}$, we have

$$
2 \pi \log \left|F_{1 n}(z) / r_{\delta}\left(F_{1 n}\right)\right| \leqq 2 \pi \log \left(r\left(F_{1 n}\right)\right) \leqq 2 \pi \log \left(r\left(F_{0 n}\right)\right) .
$$

The second inequality follows from the Corollary of Theorem 3 . We conclude that $\left\{F_{1 n}\right\}$ is bounded on any compact set $S$ and is normal. This completes the proof of Lemma 1 .

An immediate consequence of Lemma 1 is that the family $\left\{p_{h k n}\right\}$ is normal.

10. Lemma 2. If $n<n^{\prime}$, then the inequality

$$
\int_{\bar{\beta}_{n}} p d p^{*} \leqq \int_{\bar{\beta}_{n}} p d p^{*}
$$

holds for all $p \in H_{n^{\prime}}(h+k)$.

Proof. We apply the first form of Green's formula to the region 
$W_{n^{\prime}}-W_{n}$ and find

$$
\int_{\bar{\beta}_{n^{\prime}}} p d p^{*}-\int_{\bar{\beta}_{n}} p d p^{*}=D_{W_{n^{\prime}-W_{n}}}(p) \geqq 0 .
$$

Definition. $H(h+k)$ is the class of functions $p$, harmonic on $\bar{W}$, satisfying

(1) $p(z)=c_{2}(p)=$ const. for $z \in \gamma$ with $\int_{\gamma} d p^{*}=2 \pi(h+k)$,

(2) $p(\zeta)=0$,

(3) $p(z)=c_{1}(p)=$ const. for $z \in \delta$ with $\int_{\delta} d p^{*}=-2 \pi(h+k)$, and

(4) $\int_{\sigma} d p^{*}=0$ where $\sigma$ is any cycle which is homeomorphic to a circle and which does not separate $\delta$ and $\gamma$. A cycle $\sigma$ is said to separate $\delta$ and $\gamma$ if every path from $\delta$ to $\gamma$ intersects $\sigma$. Let $H$ denote the class $H(1)$.

Definition. For any $p \in H(h+k), \int_{\beta} p d p^{*}$ is understood to be $\lim _{n \rightarrow \infty} \int_{\bar{\beta}_{n}} p d p^{*}$. The existence of this limit is guaranteed by the monotonicity condition of Lemma 2.

Lemma 3. If the sequence $\left\{p_{n} ; p_{n} \in H_{n}(h+k)\right\}$ converges on compact subsets to $p^{\prime}$, then $p^{\prime} \in H(h+k)$.

We recall that a sequence $\left\{f_{n}\right\}$ converges on compact sets if for every compact set $S$, there exists an $N$ such that $\left\{f_{n} ; n \geqq N\right\}$ converges uniformly on $S$.

Proof. The convergence $p_{n} \rightarrow p^{\prime}$ is uniform on compact sets. The conditions (1), (2), and (3) for $H(h+k)$ can therefore be inferred from those of $H_{n}(h+k)$. Let $\sigma$ be any cycle which does not separate $\delta$ and $\gamma$. Then there exists $n$ such that the compact $\sigma \subset W_{n}$, and we have

$$
\sigma \sim b_{1} \delta+\sum a_{i} \beta_{i}^{\prime}
$$

where the $\beta_{i}^{\prime}$ are homologous to components of the border of $W_{n}$ (Ahlfors and Sario [1]). We embed $W_{n}$ in the complex plane with $\gamma$ as an outer boundary, and fill in the "holes" whose boundaries are the $\beta_{i}^{\prime \prime}$. Now $\sigma-b_{1} \delta=\partial A$, and every path from $\delta$ to $\gamma$ meets $\sigma$. This is a contradiction, unless $b_{1}=0$.

Using the uniform convergence of $\left\{p_{n}\right\}$ along with Green's theorem, we obtain

$$
\int_{\sigma} d p^{* *}=\lim _{n \rightarrow \infty} \int_{\sigma} d p_{n}^{*}=\lim _{n \rightarrow \infty} \int_{\sum \alpha_{i} \beta_{i}^{\prime}} d p_{n}^{*}=\lim _{n \rightarrow \infty} \sum a_{i} \int_{\beta_{i}^{\prime}} d p_{n}^{*}=0 .
$$


Definition. A harmonic function $p_{h k}$ is defined as the limit of any sequence of the normal family $\left\{p_{h k n}\right\}$ which converges on compact sets.

THEOREM 4. $p_{h k}$ minimizes the functional $\int_{\bar{\beta}} p d p^{*}-2 \pi(h-k) c(p)$ among all $p \in H(h+k)$.

The minimum value of this functional is $-2 \pi\left(h^{2} c\left(p_{0}\right)-k^{2} c\left(p_{1}\right)\right)$.

The deviation of this functional from its minimum value is $D\left(p-p_{h k}\right)$ and the minimizing function is unique.

11. There exists a subsequence $\left\{p_{n k n^{\prime}}\right\}$ of $\left\{p_{h k n}\right\}$ which converges to $p_{h k}$ on compact sets and satisfies $\lim _{n^{\prime} \rightarrow \infty} p_{h k n^{\prime}}=h p_{0}+k p_{1}$ where $p_{i}=$ $\lim _{n^{\prime} \rightarrow \infty} p_{i n^{\prime}}, i=0,1$. The uniqueness of Theorem 4 then allows us to assume that $p_{h k}=h p_{0}+k p_{1}$ for all $h$ and $k$.

Proof. That $p_{h k}$ minimizes and gives the functional the value $-2 \pi\left(h^{2} c\left(p_{0}\right)-k^{2} c\left(p_{1}\right)\right)$ will follow from Theorem 1 if we can verify (R1) - (R6) of the reduction theorem. The functionals $m_{n}$ and $m$ are taken to be $\int_{\beta_{n}} p d p^{*}-2 \pi(h-k) c(p)$ and $\int_{\bar{\beta}} p d p^{*}-2 \pi(h-k) c(p)$ respectively, while the classes $Z_{n}$ and $Z$ are $H_{n}(h+k)$ and $H(h+k)$.

If $p \in H(h+k)$, then $\left.p\right|_{w_{n}}$ satisfies the Conditions 1,2 , and 3 for $H_{n}(h+k)$. Since no $\beta_{i}\left(W_{n}\right)$ separates $\delta$ and $\gamma,\left.\int_{\beta_{i}\left(W_{n}\right)} d p\right|_{W_{n}^{*}} ^{*}=0$ and (4) is satisfied. Hence $\left.p\right|_{W_{n}} \in H_{n}(h+k)$ and (R1) is verified. The uniform convergence of $f_{k}$ to $f$ makes (R2) evident, and the functional $\int_{\bar{\beta}} p d p^{*}-$ $2 \pi(h-k) c(p)$ is defined as $\lim _{n \rightarrow \infty} \int_{\bar{\beta}_{n}} p d p^{*}-2 \pi(h-k) c(p)$, as required by (R3).

Theorem 1 shows that (R4) is satisfied, and Lemma 2 of no. 10 shows the same for (R5). That the family $\left\{p_{h k n}\right\}$ as defined in no. 2 is normal, follows from Lemma 1 of no. 9, and that the limiting functions belong to $H(h+k)$ is then a consequence of Lemma 3 of no. 10. Thus by the reduction theorem, the limit function, $p_{h k}$, minimizes the limit functional among $p \in H(h+k)$ and the minimum value of the limit functional is the limit of minimum values.

12. In order to establish the deviation formula, we first denote the functional of Theorem 1 by $\psi_{n}$ and consider its value on the function $p_{\epsilon}=p_{h k}+\epsilon\left(p-p_{h k}\right)$. Upon expanding, we find

$$
\begin{aligned}
\psi_{n}\left(p_{\epsilon}\right)= & \int_{\bar{\beta}_{n}} p_{h k} d p_{h k}^{*}-2 \pi(h-k) c\left(p_{h k}\right)+a_{1}(h) \varepsilon \\
& +\varepsilon^{2} \int_{\bar{\beta}_{n}}\left(p-p_{h k}\right) d\left(p-p_{h k}\right)^{*},
\end{aligned}
$$


where for each $n$, this is a polynomial in $\varepsilon$, and $a_{1}(n)$ is the coefficient of the $\varepsilon$ term. But the last integral is

$$
\int_{\bar{\beta}_{n}}\left(p-p_{h k}\right) d\left(p-p_{h k}\right)^{*}=\int_{\bar{\beta}_{n}+\delta+\gamma}\left(p-p_{h k}\right) d\left(p-p_{h k}\right)^{*}=D_{W_{n}}\left(p-p_{h k}\right) .
$$

The first equality follows from the fact that $p$ and $p_{h k}$ both belong to $H(h+k)$. Therefore, in the sense of limits, we write

$$
\int_{\bar{\beta}}\left(p-p_{h k}\right) d\left(p-p_{l k}\right)^{*}=D\left(p-p_{h k}\right),
$$

where $D$ is the integral over the entire bordered surface $\bar{W}$. In a similar fashion, we find

$$
\int_{\beta} p_{h k} d p_{h k}^{*}-2 \pi(h-k) c\left(p_{l k k}\right)=D\left(p_{h k}\right)-4 \pi h c\left(p_{h k}\right) .
$$

By an earlier part of this theorem, the left hand side of equation (3) is finite. Thus we have that $D\left(p_{h k}\right)<\infty$.

We assume that $D\left(p-p_{k}\right)$ is finite. By the triangle inequlity for the Dirichlet integral (Courant [1]), $D(p)$, and consequently $\int_{\beta} p d p^{*}$ are both finite. Now in equation (2), with $\varepsilon=1$, consider the limit as $n \rightarrow \infty$. The limit of every term, except $a_{1}(n)$, exists and is finite. Hence the same can be said of $\lim _{n \rightarrow \infty} a_{1}(n)$. But $\psi\left(p_{\varepsilon}\right)=\lim _{n \rightarrow \infty} \psi_{n}\left(p_{\varepsilon}\right)$ has, by part (1) of our theorem, a relative minimum for $\varepsilon=0$. Therefore, $\lim _{n \rightarrow \infty} a_{1}(n)=0$, and the deviation formula $\psi(p)=\psi\left(p_{h k}\right)+D(p-$ $p_{h k}$ ) results when $\varepsilon=1$ is substituted into equation (2) after taking limits.

When $D\left(p-p_{h k}\right)=\infty$, this formula holds in the sense that $\psi(p)=\infty$ as well. This completes the proof of Theorem 4 .

\section{Extremal and Geometric Properties of Principal Analytic Func-} tions.

Extremal properties for harmonic functions defined on a surface of finite connectivity were used in $\S 2$ to establish extremal properties of analytic functions, also defined on a surface of finite connectivity. In the present section, we exploit the extremal properties of harmonic functions, now defined on a surface of infinite connectivity, for the purpose of establishing both extremal and geometric properties of analytic functions.

13. A competing class of analytic functions is defined as follows.

Definition. $A$ is the class of analytic functions on $\bar{W}$ such that (1) $F(\gamma)$ is a circle traced once in the posititive direction, (2) $|F(\zeta)|=$ 
1, (3) $F(\delta)$ is a circle traced once in the negative direction, and (4) $F$ is univalent on $\bar{W}$.

Theorem 5. For any $F \in A, \log |F| \in H$. Furthermore $F_{i}=\exp \left(p_{i}\right.$ $\left.+i p_{i}^{*}\right) \in A, i=0,1$.

No ambiguity will result in referring also to $F_{0}$ and $F_{1}$ as principal analytic functions.

Proof. For any $F \in A$, consider $\log |F|$, which clearly satisfies (1)-(3) of the definition of $H$ in no. 10. Then let $\int_{\sigma} d(\log |F|)^{*} \neq 0$ for $\sigma$ not separating $\delta$ and $\gamma$. If $\int_{\sigma} d(\log |F|)^{*}=2 \pi k, k$ an integer, then $F(\sigma)$ separates $F(\delta)$ and $F(\gamma)$. But $F$ is univalent on $\bar{W}$ and we have the contradiction that $\sigma$ separates $\delta$ and $\gamma$. This means that $\log |F| \in H$.

Let $F_{i}=\exp \left(p_{i}+i p_{i}^{*}\right), i=0,1$. Conditions $1-3$ for $A$ are easily verified. An application of the extended argument principle to any exhausting subregion $W_{n}$ shows that $F_{i}$ is univalent on $\delta \cup \gamma$, when univalence is established at interior points. For interior points of $\bar{W}$, $F_{i}$ can be represented as $\exp \left(p_{i}+i p_{i}^{*}\right)=\lim _{n \rightarrow \infty} \exp \left(p_{i n}+i p_{2 n}^{*}\right)=\lim _{n \rightarrow \infty} F_{i n}$. So each $F_{i}$ is univalent by Theorem 2 and the well-known Hurwitz theorem.

14. The following five theorems are concerned with analytic functions constructed from the harmonic functions $p_{0}$ and $p_{1}$, w hich are uniquely defined by Theorem 4 .

Definition. $F$ is an annular radial (circular) slit mapping of $\bar{W}$ provided that $F(\bar{W})$ is an annulus minus a point set each component of which is a radial (circular) slit or point. Let $\left\{w ; r_{\delta}\left(F_{i}\right) \leqq|w| \leqq\right.$ $\left.r_{\gamma}\left(F_{i}\right)\right\}-F_{i}(\bar{W})$ be denoted by $S_{i}, i=0,1$.

DEFINITION. For a surface of infinite connectivity, the complementary logarithmic area $\mu(F)$ is defined as $\lim _{n \rightarrow \infty} \mu_{n}(F)$ for any $F \in A$. That this limit is defined independently of an exhaustion follows from Theorem 5 and Lemma 2.

THeOREM 6. $F_{0}=\exp \left(p_{0}+i p_{0}^{*}\right)$ maximizes $2 \pi \log (r(F))+\mu(F)$ among all $F \in A$.

The value of the maximum is $2 \pi \log \left(r\left(F_{0}\right)\right)$.

The deviation from the maximum is $D\left(\log |F| F_{0} \mid\right)$, and the maximizing function is unique up to a rotation.

The 2-dimensional Lebesgue measure of the point set $S_{0}$ is 0 .

$F_{0}$ is an annular radial-slit mapping. 
Proof. We apply Theorem 4 with $h=1, k=0$ and obtain that $\log \left|F_{0}\right|$ minimizes $\int_{\bar{\beta}} p d p^{*}-2 \pi c(p)$ among all $p \in H$. According to Theorem 5, we may use Theorem 4 on logarithms of functions in $A$ as well, that is, $F_{0}$ maximizes the functional $2 \pi \log (r(F))+\mu(F)$ among all $F \in A$, the maximum value of this functional is $2 \pi \log \left(r\left(F_{0}\right)\right)$, and the deviation from the maximum is $D\left(\log \left|F / F_{0}\right|\right)$.

As for the 2-dimensional Lebesgue measure of $S_{0}$, consider the annulus

$$
\left\{w ; r_{\delta}\left(F_{0}\right) \leqq|w| \leqq r_{\gamma}\left(F_{0}\right)\right\}
$$

and set $t=\log w$. The transformation mapping $w$ into $\log w$ is denoted $\mathrm{L}$, and the image of $\left\{w ; r_{\delta}\left(F_{0}\right) \leqq|w| \leqq r_{\gamma}\left(F_{0}\right)\right\}$ under $L$ is called $R$. Now it is easily seen that

$$
L S_{0}=\bigcap_{i=1}^{\infty}\left[C_{R}\left(L\left(F_{0}\left(W_{n}\right)\right)\right)\right],
$$

where $C_{R}$ is understood to mean complement with respect to $R . \quad L\left(F_{0}\left(W_{n}\right)\right)$ is compact and closed in $R$, and this means that $C_{R}\left[L\left(F_{0}\left(W_{n}\right)\right)\right]$ is open and measurable. Hence, $L S_{0}$, a countable intersection of measurable sets, is measurable. Its measure $M$ is then given by

$$
M\left(L\left(S_{0}\right)\right)=\lim _{n \rightarrow \infty} \mu_{n}\left(F_{0}\right),
$$

where $\mu_{n}\left(F_{0}\right)$ is defined in no. 7. But according to an earlier part of this theorem, the term on the right is 0 . When we observe that $L$, defined on the cut annulus, preserves sets of measure zero, we conclude that the 2-dimensional Lebesgue measure of $S_{0}$ is zero.

Suppose that the complement, with respect to $\left\{\mathrm{w} ; r_{\delta}\left(F_{0}\right) \leqq|w| \leqq\right.$ $\left.r_{\gamma}\left(F_{0}\right)\right\}$, of $F_{0}(\bar{W})$ is a point set, the components of which are not all radial slits or points. The full annulus

$$
\left\{w ; r_{\delta}\left(F_{0}\right) \leqq|w| \leqq r_{\gamma}\left(F_{0}\right)\right\}
$$

minus such a component, denoted $\eta$, is called $W_{0}$. We embed $W_{0}$ in the Riemann sphere $S^{2}$ and consider the simply connected point set $S^{2}-\eta$, which can be mapped conformally onto the complement of a unit disc. Let $E$ be this conformal mapping, and denote by $\gamma^{\prime \prime}$ and $\delta^{\prime \prime}$ the sets $E\left(\delta_{0}\right)$ and $E\left(\gamma_{0}\right)$, where $\delta_{0}=F_{0}(\delta)$ and $\gamma_{0}=F_{0}(\gamma)$. Now $E\left(W_{0}\right)$ is of finite connectivity, so we can apply Theorem 2 to construct a radial-slit mapping $\varphi$ of $E\left(W_{0}\right)$ onto an annulus, minus one radial slit, with inner boundary $\varphi\left(\delta^{\prime \prime}\right)$ and outer boundary $\varphi\left(\gamma^{\prime \prime}\right) . \quad \rho$ is normalized by $\left|\varphi \circ E_{\circ} F_{0}(\zeta)\right|=1$, and belongs to $A_{n}$ for $E\left(W_{0}\right)$. We then apply the corollary of Theorem 3 to $\varphi$ and find that $2 \pi \log (r(\varphi))>2 \pi \log \left(r\left(E^{-1}\right)\right)=2 \pi \log \left(r\left(F_{0}\right)\right)$. Then

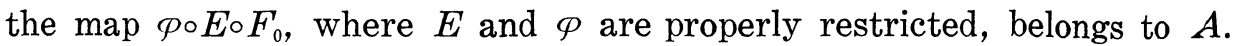
But $2 \pi \log \left(r\left(\varphi \circ E \circ F_{0}\right)\right)=2 \pi \log (r(\mathcal{P}))>2 \pi \log \left(r\left(F_{0}\right)\right)$. This is a contradic- 
tion, for according to an earlier part of this theorem $F_{0}$, up to a rotation, uniquely maximizes the functional $2 \pi \log \left(r\left(F_{0}\right)\right)$ in $A$. This completes the proof of Theorem 6 .

COROLlaRY. The principal analytic function $F_{0}$ maximizes the functional $r(F)$ among all $F \in A$.

Proof. The maximum value of the functional in Theorem 6 is $2 \pi$ $\log \left(r\left(F_{0}\right)\right)$, that is $\mu\left(F_{0}\right)=0$. The proof is complete when we observe that $\mu(F)$ is nonnegative for all $F \in A$.

Theorem 7. $F_{1}=\exp \left(p_{1}+i p_{1}^{*}\right)$ minimizes $2 \pi \log (r(F))-\mu(F)$ among all $F \in A$.

The value of the minimum is $2 \pi \log \left(r\left(F_{1}\right)\right)$.

The deviation from the minimum is $D\left(\log |F| F_{1} \mid\right)$, and the minimizing function is unique up to a rotation.

The 2-dimensional Lebesgue measure of the point set $S_{1}$ is zero.

$F_{1}$ is an annular circular-slit mapping.

The proof is analogous to that of Theorem 6 and uses $h=0, k=1$.

CoRollary. The principal analytic function $F_{1}$ minimizes the functional $r(F)$ among all $F \in A$.

TheOREM 8. $P=\sqrt{F_{0} \cdot F_{1}}$ maximizes $\mu(F)$ among all $F \in A$.

The value of the maximum is $\mu(P)$.

The deviation from the maximum is $D(\log |F| P \mid)$, and the maximizing function is unique up to a rotation.

The proof uses $h=1 / 2, k=1 / 2$.

Theorem 9. $Q=F_{0} \mid F_{1}$ maximizes $4 \pi \log (r(F))-D(\log |F|)$ among all quotients of functions in $A$.

The value of the maximum is $2 \pi \log (r(Q))$.

The deviation from the maximum is $D(\log |F / Q|)$.

Proof. When the condition $h=1, k=-1$ is substituted into Theorem 4 , it is easily seen that the technique of Theorem 3 will establish Theorem 9 .

Consider the multiple-valued functions $P_{0}=p_{0}+i p_{0}^{*}$ and $P_{1}=p_{1}+$ $i p_{1}^{*}$. The difference of these functions has zero flux around any cycle of $\bar{W}$ and is single-valued.

Theorem $10, P_{0}-P_{1}$ maximizes 


$$
4 \pi\left[\operatorname{Re}\left(F\left(z_{2}\right)-F\left(z_{1}\right)\right)\right]-D(F)
$$

among all analytic functions on $\bar{W}$ the real part of which is constant on $\delta$, constant on $\gamma$, and 0 at $\zeta$. Here $z_{1}$, and $z_{2}$ are on $\gamma$ and $\delta$ respectively.

The value of the maximum is $-2 \pi \operatorname{Re}\left[\left(P_{0}-P_{1}\right)\left(z_{2}\right)-\left(P_{0}-P_{1}\right)\left(z_{1}\right)\right]$.

The deviation from the maximum is $D\left(F-\left(P_{0}-P_{1}\right)\right)$.

The proof again applies Theorem 4 , with $h=1$ and $k=-1$, as well as the observation that $\operatorname{Re}\left(P_{0}-P_{1}\right)=p_{0}-p_{1}$ and $R e F \in H$ when $F$ is analytic on $\bar{W}$.

\section{The Existence of Distinct Principal Analytic Functions.}

15. We consider the problem of determining conditions under which there exist two different principal analytic functions on the planar bordered Riemann surface $\bar{W}$ of no. 1 . The principal analytic functions under consideration are defined in no. 13, and have properties described in Theorems 5, 6, and 7 of no. 14. The following concepts are dealt with in Ahlfors and Sario [1].

Definition. Two compact sets in the plane, each with connected complement, are said to be equivalent if their complements are conformally equivalent.

For the remainder of this chapter, we let $E$ be a compact plane set with connected complement.

Theorem (Ahlfors and Sario [1]). The complement of $E$ is of class $0_{A D}$ if and only if every set which is equivalent to $E$ has 2-dimensional Lebesgue measure 0.

DEFinition. Let $U$ be any open set which contains $E$, and suppose that a function $F$ is analytic on $U-E . \quad E$ is said to be a removable singularity for $F$ if there exists analytic extension of $F$ to $U$.

Theorem (Ahlfors and Sario [1]). E is a removable singularity for all functions of class $A D$ in a neighborhood of $E$ if and only if the complement of $E$ (with respect to the Riemann sphere) is of class $0_{A}$ D.

16. Definition. A planar bordered Riemann surface $\bar{W}$ as described in no. 1 is said to have rigid radius when $r(F)$ is constant for every $F$ in the class $A$ of no. 13.

THEOREM 11, Let $F_{9}$ and $F_{7}$ be the principal analytic functions 
belonging to $A$. The surface $\bar{W}$ has rigid radius if and only if $F_{0}=$ $c F_{1}$, where $|c|=1$.

Proof. If $\bar{W}$ has rigid radius, then according to Theorems 6 and 7 , both $F_{0}$ and $F_{1}$ minimize the same functional. Hence $F_{0}=c F_{1}$, with $|c|=1$. On the other hand, if $F_{0}=c F_{1}$, we conclude from the corollaries of Theorems 6 and 7 that $F_{0}$ maximizes, and $F_{1}$ minimizes the functional $r(F)$ among all $F \in A$. Because $|c|=1$, we have that the radius is rigid.

\section{AD-Removability.}

17. Our next condition for distinguishing $F_{0}$ from $F_{1}$ is most naturally stated if we take the bordered Riemann surface $\bar{W}$ to be a plane region, with $\gamma$ and $\delta$ as outer and inner boundaries respectively. In addition, we let $\bar{W}_{1}$ denote the plane point set bounded by $\gamma$ and $\delta$, with $E$ the difference $\bar{W}_{1}-\bar{W}$.

THEOREM 12. Let $F_{0}$ and $F_{1}$ be the principal analytic functions of no. 13. Then $F_{0}=c F_{1}$, with $|c|=1$, if and only if $S^{2}-E \in 0_{A D}$.

Sufficiency. $\quad F_{0}$ and $F_{1}$ map a neighborhood $U$ of $E$ onto an open set of finite area and are of class $A D$ in this neighborhood of $E$. Then according to no. 15, the principal analytic functions may be extended to all of $\bar{W}$. If the extension $F_{i}$ of $F_{i}$ satisfies $\widetilde{F}_{i}\left(z_{0}\right)=w_{0}$ for some $w_{0}$ with $r_{\delta}\left(\widetilde{F}_{i}\right)<\left|w_{0}\right|<r_{\gamma}\left(\widetilde{F}_{i}\right)$, then

$$
(2 \pi i)^{-1} \int_{\delta+\gamma} \frac{d \widetilde{F}_{i}}{\widetilde{F}_{i}-w_{0}}=(2 \pi i)^{-1} \int_{\delta+\gamma} \frac{d F_{i}}{F_{i}-w_{0}}=1, \quad i=0,1 .
$$

Since $F_{i} \in A$, the second integral is 1 and the extensions are univalent. This means that $\widetilde{F}_{1} \circ \widetilde{F}_{0}^{-1}$ is a conformal mapping of a full closed annulus, and in fact that $r\left(F_{0}\right)$ is equal to $r\left(F_{1}\right)$. We have $F_{0}=c F_{1}$, with $|c|=1$, as a consequence of Theorem 11.

Necessity. If $S^{2}-E$ is not of class $0_{A D}$, then, according to no. 15, there exists a one to one conformal mapping with positive complementary area. Such a mapping will have positive complimentary logarithmic area as well. Therefore, according to Theorem $8, \mu\left(\sqrt{F_{0} \cdot F_{1}}\right)$ is positive, and Theorem 6 guarantees that $F_{0} \neq c F_{1}$.

\section{APPENDIX}

An argument of Ahlfors and Beurling [1] (p.111), which will be referred to and not repeated, is crucial in the proof of:

18. THEOREM 11. The analytic function $P_{n}=\sqrt{\overline{F_{0 n} \cdot F_{1 n}}}$ is of 
class $A_{n}$.

Proof. Verification of the Conditions 1,2, and 3 for $A_{n}$ of no. 5 is immediate. Only (4) remains to be checked. If $\log F_{1 n}$ and $\log F_{0 n}$ are considered in the roles of $q$ and $p$ of Ahlfors and Beurling [1], p. 111, then $\log \sqrt{F_{0 n} \cdot F_{1 n}}$ may be considered in the role of $\frac{1}{2}(q+p)$. We observe that $d\left(\log F_{1 n}\right) / d\left(\log F_{0 n}\right)$ is well defined on the approximating $W_{n}$. Hence, by the technique of Ahlfors and Beurling already cited, we may conclude that $R e\left(d \log F_{1 n} / d \log F_{0 n}\right)$ is of constant sign with no zeros in $W_{n}$. This implies that the image of each contour $\beta_{i}$ is a convex curve, and each image is traced once as each $\beta_{i}$ is traced once. This also implies that each of the curves $F\left(\beta_{\imath}\right)$ is traced in the same direction, and this direction will be determined now for one $F\left(\beta_{i}\right)$.

We observe that for each $i, P_{n}\left(\beta_{\imath}\right)$ is a compact set, and we may then choose $w_{i}$ and $w_{i}^{\prime}$ so that $w_{\imath}$ is that point of $P_{n}\left(\beta_{i}\right)$ which is closest to $P_{n}(\gamma)$ and $w_{i}^{\prime}$ is that point of $P_{n}(\gamma)$ which is closest to $P_{n}\left(\beta_{i}\right)$. We now assume that the $\beta_{i}$ are indexed so that $\min \left\{d\left(w_{i}, w_{i}^{\prime}\right) ; i=1,2, \cdots\right.$, $k(n)\}$ is $d\left(w_{1}, w_{1}^{\prime}\right)$ where $d\left(w, w^{\prime}\right)$ is the usual Euclidean distance from $w$ to $w^{\prime}$. That is to say, $P_{n}\left(\beta_{1}\right)$ is as close to $P_{n}(\gamma)$ as any of $P_{n}\left(\beta_{2}\right), \cdots$, $P_{n}\left(\beta_{k(n)}\right)$. The line segment $I^{\prime}$ joining $w_{1}$ to $w_{1}^{\prime}$ is a univalence path for $P_{n}$ in the sense that each point of $\Gamma$ is taken exactly once by a point

of $W_{n}$. Clearly $P_{n}$ is one to one on $P_{n}^{-1} \Gamma$, and we may conclude that $\beta_{1}$ and $P_{n}\left(\beta_{1}\right)$ are similarly oriented. The reasoning in the paragraph above then establishes that each $P_{n}\left(\beta_{i}\right)$ is oriented as is $P_{n}\left(\beta_{1}\right)$, and in fact, for each $i$ we have that the winding number for points inside $P_{n}\left(\beta_{i}\right)$ is -1 .

An application of the argument principle is now all that is needed to show that $P_{n}$ is univalent on Int $W_{n} \cup \delta \cup \gamma$.

\section{BIBLIOGRAPHY}

1. L. Ahlfors, [1] Complex analysis, McGraw-Hill Book Company, Inc., (1952).

2. L. Ahlfors, and A. Beurling, [1] Conformal invariants and function-theoretic null-sets, Acta Math., 83 (1950), 101-129.

3. L. Ahlfors, and L. Sario, [1]Riemann surfacees, Princeton University Press, (1960).

4. R. Courant, [1] Dirichlet's principle, conformal mapping, and minimal surfaces, Interscience Publishers (1950), 13-14.

5. R. de Possel, [1] Sur quelpues propriétés de la representation conforme des domains multiplement connexes, en relation avec le théorème des fentes parallèles, Math. Ann., 107 (1932), 496-504.

6. L. Sario, [1] Ueber Riemannsche Flächen mit hebbarem Rand, Ann. Acad. Sci. Fenn. Ser. A. I. no. 50 (1948), 79 pp.

7. - [2] A linear operator method on arbitrary Riemann surfaces, Trans. Amer. Math. Soc., 72 (1952), 281-295.

8. _ [3] Strong and weak boundary components, J. Analyse Math. 5 (1956/57), 389398.

9. M. Schiffer, [1] Some recent developments in the theory of conformal mapping, Appendix to $R$. Courant, Dirichlet's principle, conformal mapping, and minimal surfaces, Interscience Publishers (1950), 249-265. 



\section{PACIFIC JOURNAL OF MATHEMATICS}

\section{EDITORS}

\author{
RaLPh S. Phillips \\ Stanford University \\ Stanford, California \\ F. H. BRowNELL \\ University of Washington \\ Seattle 5 , Washington
}

A. L. Whiteman

University of Southern California

Los Angeles 7, California

L. J. Paige

University of California

Los Angeles 24, California

\author{
E. F. BECKENBACH \\ T. M. CHERRY
}

\author{
ASSOCIATE EDITORS

$\begin{array}{lll}\text { D. DERRY } & \text { H. L. ROYDEN } & \text { E. G. STRAUS } \\ \text { M. OHTSUKA } & \text { E. SPANIER } & \text { F. WOLF }\end{array}$

\section{SUPPORTING INSTITUTIONS}

\author{
UNIVERSITY OF BRITISH COLUMBIA \\ CALIFORNIA INSTITUTE OF TECHNOLOGY \\ UNIVERSITY OF CALIFORNIA \\ MONTANA STATE UNIVERSITY \\ UNIVERSITY OF NEVADA \\ NEW MEXICO STATE UNIVERSITY \\ OREGON STATE COLLEGE \\ UNIVERSITY OF OREGON \\ OSAKA UNIVERSITY \\ UNIVERSITY OF SOUTHERN CALIFORNIA
}

\author{
STANFORD UNIVERSITY \\ UNIVERSITY OF TOKYO \\ UNIVERSITY OF UTAH \\ WASHINGTON STATE COLLEGE \\ UNIVERSITY OF WASHINGTON \\ AMERICAN MATHEMATICAL SOCIETY \\ CALIFORNIA RESEARCH CORPORATION \\ HUGHES AIRCRAFT COMPANY \\ SPACE TECHNOLOGY LABORATORIES \\ NAVAL ORDNANCE TEST STATION
}

Mathematical papers intended for publication in the Pacific Journal of Mathematics should be typewritten (double spaced), and the author should keep a complete copy. Manuscripts may be sent to any one of the four editors. All other communications to the editors should be addressed to the managing editor, L. J. Paige at the University of California, Los Angeles 24, California.

50 reprints per author of each article are furnished free of charge; additional copies may be obtained at cost in multiples of 50 .

The Pacific Journal of Mathematics is published quarterly, in March, June, September, and December. The price per volume (4 numbers) is $\$ 12.00$; single issues, $\$ 3.50$. Back numbers are available. Special price to individual faculty members of supporting institutions and to individual members of the American Mathematical Society: $\$ 4.00$ per volume; single issues, $\$ 1.25$.

Subscriptions, orders for back numbers, and changes of address should be sent to Pacific Journal of Mathematics, 103 Highland Boulevard, Berkeley 8, California.

Printed at Kokusai Bunken Insatsusha (International Academic Printing Co., Ltd.), No. 6, 2-chome, Fujimi-cho, Chiyoda-ku, Tokyo, Japan.

\section{PUBLISHED BY PACIFIC JOURNAL OF MATHEMATICS, A NON-PROFIT CORPORATION}

The Supporting Institutions listed above contribute to the cost of publication of this Journal, but they are not owners or publishers and have no responsibility for its content or policies.

Reprinted 1966 in the United States of America 


\section{Pacific Journal of Mathematics}

\section{Vol. 11, No. 4}

A. V. Balakrishnan, Prediction theory for Markoff processes . . . . . . . . . . 1171

Dallas O. Banks, Upper bounds for the eigenvalues of some vibrating systems . . . . 1183

A. Białynicki-Birula, On the field of rational functions of algebraic groups ...... 1205

Thomas Andrew Brown, Simple paths on convex polyhedra .............. 1211

L. Carlitz, Some congruences for the Bell polynomials . . . . . . . . . . . . 1215

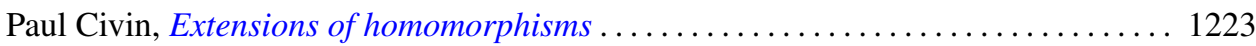

Paul Joseph Cohen and Milton Lees, Asymptotic decay of solutions of differential

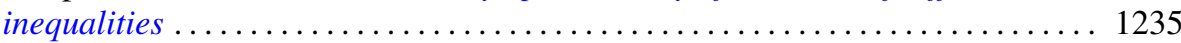

István Fáry, Self-intersection of a sphere on a complex quadric . . . . . . . . . . 1251

Walter Feit and John Griggs Thompson, Groups which have a faithful representation

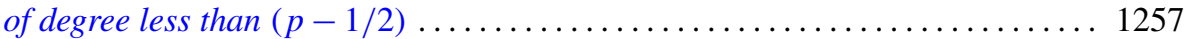

William James Firey, Mean cross-section measures of harmonic means of convex

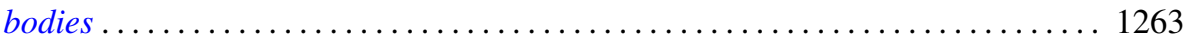

Avner Friedman, The wave equation for differential forms . . . . . . . . . . 1267

Bernard Russel Gelbaum and Jesus Gil De Lamadrid, Bases of tensor products of

Banach spaces ................................... 1281

Ronald Kay Getoor, Infinitely divisible probabilities on the hyperbolic plane . . . . 1287

Basil Gordon, Sequences in groups with distinct partial products . . . . . . . . . . . . 1309

Magnus R. Hestenes, Relative self-adjoint operators in Hilbert space . . . . . . . . . 1315

Fu Cheng Hsiang, On a theorem of Fejér ......................... 1359

John McCormick Irwin and Elbert A. Walker, On N-high subgroups of Abelian

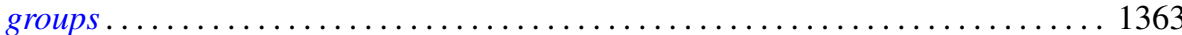

John McCormick Irwin, High subgroups of Abelian torsion groups . . . . . . . . . 1375

R. E. Johnson, Quotient rings of rings with zero singular ideal . . . . . . . . . . . 1385

David G. Kendall and John Leonard Mott, The asymptotic distribution of the time-to-escape for comets strongly bound to the solar system ...

Kurt Kreith, The spectrum of singular self-adjoint elliptic operators ....

Lionello Lombardi, The semicontinuity of the most general integral of the calculus of variations in non-parametric form ................................

Albert W. Marshall and Ingram Olkin, Game theoretic proof that Chebyshev inequalities are sharp

Wallace Smith Martindale, III, Primitive algebras with involution . . William H. Mills, Decomposition of holomorphs ..............

James Donald Monk, On the representation theory for cylindric algebras . . . . . . 1447

Shu-Teh Chen Moy, A note on generalizations of Shannon-McMillan theorem . . . . 1459

Donald Earl Myers, An imbedding space for Schwartz distributions . .

John R. Myhill, Category methods in recursion theory .........

Paul Adrian Nickel, On extremal properties for annular radial and circular slit mappings of bordered Riemann surfaces

Edward Scott O'Keefe, Primal clusters of two-element algebras . .

Nelson Onuchic, Applications of the topological method of Wazewski to certain

problems of asymptotic behavior in ordinary differential equations ...

Peter Perkins, A theorem on regular matrices................

Clinton M. Petty, Centroid surfaces .... 